\title{
An outline of type-theoretical approaches to lexical semantics
}

\author{
Robin Cooper ${ }^{1}$ and Christian Retoré ${ }^{2}$ \\ ${ }^{1}$ University of Gothenburg \\ ${ }^{2}$ Université de Montpellier \& LIRMM
}

\begin{abstract}
We take the opportunity of the publication of some of the papers of the ESSLLI workshop TYTLES (TYpe Theory and LExical Semantics, ESSLLI 2015, Barcelona) to provide an overview of the possibilities that type theory offers to model lexical semantics, especially the type-theoretical frameworks that properly model compositional

Keywords: lexical semantics; compositional semantics; type theory; lambda calculus
\end{abstract} semantics.

ORIGINS OF THIS ISSUE: ESSLLI WORKSHOP ON
TYPE THEORY AND LEXICAL SEMANTICS (2015)

The program of the ESSLLI 2015 workshop held in Barcelona ${ }^{1}$ consisted of twelve selected talks. The corresponding extended abstracts, together with an introduction and a conclusion by the workshop organisers, are available on the web as Cooper and Retoré (2015); it includes:

A. Introduction (slides), by Robin Cooper and Christian Retoré.

B. Justyna Grudzińska and Marek Zawadowski. A Puzzle about Longdistance Indefinites and Dependent Type Semantics.

C. Stergios Chatzikyriakidis, Mathieu Lafourcade, Lionel Ramadier and Manel Zarrouk. Type Theories and Lexical Networks: Using Serious Games as the Basis for Multi-Sorted Typed Systems.

\footnotetext{
${ }^{1}$ On 17 August 2017, while writing this introduction, we learnt about the tragic attack in Barcelona, where some friends and colleagues live. We would like to express our sympathy.
} 
D. Staffan Larsson. Perceptual Meaning in TTR Judgement-based Semantics and Conceptual Spaces.

E. Simon Dobnik. Interfacing Language, Spatial Perception and Cognition in Type Theory with Records.

F. Peter Sutton and Hana Filip. Probabilistic Mereological TTR and the Mass/Count Distinction.

G. Ellen Breitholtz. Are Widows Always Wicked? Learning Concepts through Enthymematic Reasoning.

H. Bruno Mery. The Relative Complexity of Constraints in Co-Predicative Utterances.

I. Daisuke Bekki and Miho Satoh. Calculating Projections via Type Checking.

J. Laura Kallmeyer, Timm Lichte, Rainer Osswald, Sylvain Pogodalla and Christian Wurm. Quantification in Frame Semantics with Hybrid Logic.

K. Livy Real and Alexandre Rademaker. An Overview on Portuguese Nominalisation.

L. Pepijn Kokke. Formalising type-logical grammars in Agda.

M. Seohyun Im and Chungmin Lee. A Developed Analysis of Type Coercion Using Asher's TCL and Conventionality.

N. Ribeka Tanaka, Koji Mineshima and Daisuke Bekki. Factivity and Presupposition in Dependent Type Semantics.

O. Conclusion (slides), by Robin Cooper and Christian Retoré.

Some of these papers were submitted and some of these are now included in this issue of the Journal of Language Modelling on Type theory and lexical semantics.

Let us briefly present this fruitful connection of lasting interest.

1

\section{A GOMPOSITIONAL VIEW}

\section{OF LEXICAL SEMANTICS}

The relation between lexical semantics and type theory is rather unnatural if one thinks of lexical semantics as defined, e.g., in the article Lexical Semantics in the Oxford Research Encyclopedia of Linguistics (Geeraerts 2017): 
Lexical semantics is the study of word meaning. Descriptively speaking, the main topics studied within lexical semantics involve either the internal semantic structure of words, or the semantic relations that occur within the vocabulary. Within the first set, major phenomena include polysemy (in contrast with vagueness), metonymy, metaphor, and prototypicality. Within the second set, dominant topics include lexical fields, lexical relations, conceptual metaphor and metonymy, and frames.

If we have a look from the other side, i.e., philosophy of language, where logic, compositional semantics and type theory live, the connection is at least evoked as in these words from the entry on Word Meaning in the Stanford Encyclopaedia of Philosophy (Gasparri and Marconi 2016):

Word meaning has played a somewhat marginal role in early contemporary philosophy of language, which was primarily concerned with the structural features of sentences and showed less interest in the format of lexical representations and in the nature of the word-level input to compositional processes. Nowadays, it is well-established that the way we account for word meaning is bound to have a major impact in tipping the balance in favor or against a given picture of the fundamental properties of human language. This entry provides an overview of the way issues related to lexical meaning have been explored in analytic philosophy and a summary of relevant research on the subject in neighboring scientific domains.

So this survey, as well as the workshop, is devoted to the study of lexical semantics in a compositional framework deriving - roughly speaking - from Montague semantics and the lexical issues to be dealt with are:

- word meaning in context (various forms of polysemy),

- relation between meanings,

- relation between lexical networks and lexical semantics.

Observe that from a logical viewpoint, relations between meanings are naturally higher order relations which oblige us to go beyond 
first order logic and type theories are naturally higher order - of course reification à la Davidson is possible, but still rather unnatural and less compositional.

Usual techniques for taking into account at least part of lexical semantics are the descriptions using features (e.g. human/non-human), argument structures which specify the nature of arguments to predicates and the composition of word vectors that has been quite fashionable recently although it obliges us to leave out some of the logical structure involved in compositional semantics such as negation.

2

SOME ASPEGTS OF LEXICAL SEMANTIGS IN GOMPOSITIONAL FRAMEWORKS

2.1

Polysemy

Polysemy is the phenomenon that a single word or expression has several readings. It is common to distinguish various forms of polysemy.

Simple polysemy might be viewed as the coincidence that a word has several unrelated meanings, which in some contexts may be hard to choose between, as in (1).

(1) a. The river flowed by the bank.

b. The bank is near the river.

c. The bank phoned me.

This should be distinguished from words that have several interrelated meanings derived from a root meaning. An institution like a journal or a town have such aspects, which are also called facets, as shown in (2).

a. The journal is printed on pink paper.

b. The journal hired a new commentator.

c. The journal is near the port.

Events are a special case of this, and they play a particular role in semantics. Deverbals may refer to aspects of an action verb such as the process, the result, the place or the material used, as in (3)-(4). There is a rich literature on the topic, see, e.g., the references in Real and Retoré (2014).

(3) a. The signature took three months.

b. The signature is unreadable. 
(4) a. The building in front of my house took three months.

b. The building in front of my house is ugly.

There is a special form of polysemy where the two aspects are strongly linked: one aspect does not exclude the other, on the contrary you cannot have one without the other. This has some consequences for the individuation process and the interpretation of the quantifiers, as shown in (5).

(5) a. I carried all the books from the library to the attic.

b. I read all the books in the library.

There are examples that are hard to understand without the context, which can be linguistic or extralinguistic, as in (6).

(6) a. I am parked behind a blue BMW.

b. The ham sandwich asked for a coffee.

Co-predication

Given that compositional semantics is quite interested in the logical structure of sentences, it is normal that it has been studying how one can conjoin the properties of a word, properties which may concern only a single aspect of this word, as in (7)-(10).

(7) Dinner was delicious but took ages. (event/food)

(8) * The salmon we had for lunch was lightning fast. (animal/food)

(9) a. I left my preferred book on logic on the table. (physical/information)

b. I carried the books from the shelf to the attic since I already read them. (physical/information)

(10) a. Liverpool is a poor town and an important harbour. (people/geographic)

b.* Liverpool defeated Chelsea and is an important harbour. (football/geographic)

It can be observed that in some thematic contexts or contrasts a priori infelicitous co-predication may become felicitous.

(11) a. Barcelona won four champions leagues and organised the olympiads. 
b. Libourne, a small south-west town, defeated Lille.

Deverbals rarely allow co-predications on their different facets, as discussed in Real and Retore (2014) and at the workshop, in Livy Real's talk.

\section{INTEGRATING LEXICAL SEMANTICS INTO A GOMPOSITIONAL AND GOMPUTATIONAL FRAMEWORK}

Standard lexical semantics, including distributional semantics as used in natural language processing, involving big data, machine learning, information retrieval, and so on, is mainly concerned with what a sentence or a text is about in terms of empirically grounded word meanings. For instance, word vectors are derived from the cooccurrences of words in texts. They are especially good for the study of semantic similarity: the cosine measure of similarity or the products of vectors by matrices may model the combination of a verb and its object or of an adjective with a noun, etc.

Formal semantics is rather concerned with logical and pragmatic relations: what a sentence (or discourse) asserts, denies, supposes, how noun phrases and pronouns (co)refer to individuals and sets, in which situations (or worlds) sentences are true. It is also concerned with the interpretation of modality, aspect and tense. Usually intepretation assumes that lexical meaning has been determined in some way external to the semantics. It is carried out in two steps: word meanings are combined according to syntactic analysis into a logical formula, which is thereafter interpreted in terms of some semantics, usually possible worlds semantics, although other interpretations of logical formulas are possible, like situation semantics or game-theoretical semantics.

These two approaches are complementary, and an adequate theory of semantics should take both into account. For instance, if one is looking for an answer to the question whether Geach was a student of Wittgenstein, one can find in the French Wikipedia (contradicting the English version):

(12) In 1941, [Geach] married Elisabeth Anscombe, through whom he got in contact with Wittgenstein. Although he never attended the lectures of the latter, he was strongly influenced by him. 
Both word meaning ("student of", "to attend some lectures by") and sentence/discourse structure are needed to understand this text (scope of the negative "never", reference of pronouns like "he", "whom", "the latter", "his" and "him").

In Human Machine Interaction large scale analysis on the fly is not practical but proper understanding is still important. For instance, if a parent says "the children want pizza" to some McDonald's-like automaton, the person who treats the order needs to know whether to prepare one or several pizzas and in this case the system should know that this has not been determined by the utterance and should therefore ask for a clarification.

\subsection{Pustejovsky's generative lexicon: a framework for polysemy}

Important and pioneering work on polysemy has been carried out by Pustejovsky. Although those questions have been studied at least since the 1970s (Apresjan 1974; Bierwisch 1979, 1983; Nunberg 1979, 1995; Cruse 1986; see, e.g., Lauer 2004 or Dölling 2018 for survey and comparisons), Pustejovsky (1991, 1995) was the first to propose a formal compositional framework for handling word meaning and the transformation of word meaning in context.

The basic components of Pustejovsky's approach are:

- a compositional (generative) view of word meaning,

- a formal framework: word meaning as complex feature structures (he way they combine is less specified),

- computational tractability.

There are four levels in an entry of the generative lexicon:

- lexical typing structure: giving an explicit type for a word positioned within a type system for the language,

- argument structure: specifying the number and nature of arguments to a predicate,

- event structure: defining the event type of the expression and any subevent structure it may have,

- qualia structure: a structural differentiation of the predicative force for a lexical item organised in four quale:

- formal: the basic category which distinguishes the meaning of a word within a larger domain, 
- constitutive: the relation between an object and its constituent parts,

- telic: the purpose or function of the object, if there is one,

- agentive: the factors involved in the object's origins or "coming into being".

Types play an important role in the generative lexicon. There are base types organised as an ontology. Functional types are also used, in particular, in the argument structure.

To sum up, the generative lexicon is the first compositional semantic framework that integrates some aspects of lexical meaning. Some of the structures and notions involved in this framework are fully formalised, but not all of them. For instance, the structure of the entries is completely formalised. Nevertheless some aspects remain to be made precise, such as the set of base types and their ontology. When it comes to the way lexical items combine, the composition modes and rules are mainly described in terms of examples, whereas automated semantic analysis would require a general procedure as well as a precise correspondence between syntactic operations and semantic rules. So one may wonder whether this framework is already able to compute the semantics of a whole complex sentence, or of a small discourse.

It is worth noticing that some important parts of the generative lexicon can be learnt, in particular the qualia structure (Claveau et al. 2003). It is still an open question whether other fields than qualia structure can be learnt.

\section{Lexical semantics and compositionality} Selectional restrictions

One way to start addressing lexical issues in compositional semantics concerns selectional restrictions, as in (13).

(13) The chair barked.

(14) Dictionary: "barks" is said of an animal, usually a dog.

A commonly adopted idea is that infelicitous semantic composition is a type mismatch: a predicate $P$ over $A$ entities (a function from objects of type $A$ to propositions or truth values) is applied to an entity $t$ of type $B$ with $B \neq A$ : 


$$
P^{A \rightarrow \operatorname{prop}}\left(t^{B}\right)
$$

as it is the case in example (13):

$$
\text { Bark }^{\text {animal } \rightarrow \text { prop }}(\text { the chair })^{\text {physical object }}
$$

This constraint needs to be relaxed in certain contexts. While (13) sounds strange, (15a) is much easier to interpret and (15b) provides a naturally occurring example.

(15) a. I was so late for registration that the secretary barked at me.

b. Bow Wow barked at on Twitter for claiming he flies private (https://www.cnet.com/news/bow-wow-barked-at-ontwitter-for-claiming-he-flies-private/, 27/8/2017)

Observe that meaning transfers are idiosyncratic. In French you can say that either a tyre or a car is "punctured", as in (16a) and (16b). Correspondingly, in English you can say that a tyre or a car has a puncture, as in (16c). However, while you can say that a tyre is flat or punctured, as in (16d), in English you cannot say that a car is flat or punctured, as in (16e).

(16) a. Le pneu est crevé.

The tyre is punctured.

b. Ma voiture est crevée.

My car is punctured.

My car has a puncture.

c. My tyre/car has a puncture.

d. My tyre is flat/punctured.

e.\#My car is flat/punctured.

Idiosyncratic phenomena can even be observed in the same language. Indeed some words with the same "ontological type" may have different meanings. For instance in French, of two words designating a set of students, namely classe (class/classroom) and promotion (year group) only classe may mean classroom.

a. La classe de CP a été repeinte pendant les The class of 1st-year was repainted during the vacances.

holidays. 
b. \#La promotion 2015 a été repeinte pendant les The year-group 2015 was repainted during the vacances.

holidays.

One issue is whether adaptation of word meaning to context should be word-driven or type-driven. A related issue is whether the base types should be related to ontological classes or to linguistic behaviours, different answers being developed by Asher (2011), Retoré (2014), Kinoshita et al. (2017), Chatzikyriakidis and Luo (2017), Mery and Retoré (2017).

There are three broad areas relating to the lexicon represented in the papers here which suggest that a type-theoretical approach can be useful. These are:

- dynamic aspects of the lexicon,

- use of dependent types,

- probability.

We will discuss each of these in turn.

4.1

Dynamic aspects of the lexicon

There is general agreement in these papers that lexical meaning is to be treated dynamically. This idea relates, of course, to the original work on the generative lexicon and notions of coercion. But it also relates to the fact that we are constantly learning new words and meanings for words, that lexical meaning is in flux.

In type-theoretical approaches there is a focus on the types of objects rather than the sets of objects (witnesses or inhabitants) which belong to those types. In introducing types we attempt to define the conditions under which an object would belong to the type rather than simply associating a set of objects with the type. This means that we can adapt a type theory to models where the set of witnesses of a type may change dynamically over time, without the type itself thereby changing. It introduces the possibility of modelling how we observe new witnesses of a type as we discover more of the world. This is 
different from a Montagovian notion of sense: a function from possible worlds and times to extensions. If you discover a new object at a given world and time, then the Montagovian sense is different.

Another dynamic aspect offered by a type theory is that the type associated with a word can change over time. Many modern type theories offer a notion of structured types (such as record types) which allows us to give an account of change not available in a Montagovian sense. For example, a record type can be changed by adding or removing a field whereas in a Montagovian sense the only structure we have is that of the set of ordered pairs which is the function from world-time pairs to extensions.

Papers relating to some kind of dynamic aspect of types in this issue are those by Chatzikyriakidis et al. and Dobnik et al.

The notion of structured type figures indirectly in the paper by Kallmeyer et al. The notion of frame which they introduce in terms of hybrid logic relates intuitively to the notion of frame in terms of record types discussed in Cooper (2016). It would be interesting, for example, to explore whether the expressions of hybrid logic can be thought of as record types modelling event types.

Dependent types are parametrised types which return a type depending on what objects are provided for their parameters. They can be thought of a functions from objects to types. A classical use of dependent types is for donkey anaphora, as first presented in Sundholm (1986) and discussed in Ranta (1994). However, a number of other uses have been pointed out in the literature. In the papers in this issue their use is discussed for presupposition (Tanaka et al.).

\section{Probability}

In standard type theory judgements that objects are of a given type are categorical: either an object is of a type or it is not. However, it seems intuitive that agents make probabilistic judgements: it is probable that a given object is of a given type, but it is not certain. Cooper et al. (2015) proposed a probabilistic type theory that could be used for natural language semantics and in this issue Sutton et al. apply this to the analysis of the mass/count distinction. 
This special issue represents a broad span of approaches using different type theories but, as we have tried to point out in this introduction, they share a number of common assumptions and goals. This bodes well for future research on type theory and lexical semantics.

\section{REFERENGES}

Juri APRESJAN (1974), Regular Polysemy, Linguistics, 14:5-32.

Nicholas AsHer (2011), Lexical Meaning in context - a web of words, Cambridge University press.

Manfred BIERWISCH (1979), Wörtliche Bedeutung - eine pragmatische Gretchenfrage, in G. GREWENDORF, editor, Sprechakttheorie und Semantik, pp. 119-148, Surkamp, Frankfurt.

Manfred BIERWISCH (1983), Semantische und konzeptuelle Repräsentation lexikalischer Einheiten, in R. RÜŽIČKA and W. MOTSCH, editors, Untersuchungen zur Semantik, pp. 61-99, Akademie-Verlag, Berlin.

Stergios CHATZIKYRIAKIDIS and Zhaohui LUO (2017), On the Interpretation of Common Nouns: Types Versus Predicates, in Stergios CHATZIKYRIAKIDIS and Zhaohui Luo, editors, Modern Perspectives in Type Theoretical Semantics, pp. 43-70, Springer.

Vincent Claveau, Pascale SÉBillot, Cécile FABre, and Pierrette Bouillon (2003), Learning Semantic Lexicons from a Part-of-Speech and Semantically Tagged Corpus Using Inductive Logic Programming, Journal of Machine Learning Research, 4:493-525.

Robin COOPER (2016), Frames as Records, in Annie FORET, Glyn MORRILL, Reinhard Muskens, Rainer OsswALD, and Sylvain PogodAlla, editors, Formal Grammar: 20th and 21st International Conferences FG 2015, Barcelona, Spain, August 2015, Revised Selected Papers FG 2016, Bozen, Italy, August 2016, Proceedings, number 9804 in LNCS, pp. 3-18, Springer.

Robin CoOper, Simon DobNik, Shalom LAPPIN, and Staffan LARSSON (2015), Probabilistic Type Theory and Natural Language Semantics, Linguistic Issues in Language Technology, 10(4):1-45.

Robin COOPER and Christian RETORÉ (2015), Extended abstracts of the ESSLLI 2015 workshop TYTLES: Types Theory and Lexical Semantics, HAL Archives Ouvertes, https://hal.archives-ouvertes.fr/hal-01584832.

D.A. CRUSE (1986), Lexical semantics, Cambridge textbooks in linguistics, Cambridge University Press, ISBN 9780521276436, http: //books.google.fr/books?id=xDSBaet2uSsC. 
Johannes DöLling (2018), Systematic polysemy, in Lisa MATTHEwson, Cécile Meier, Hotze Rullmann, and Thomas Ede Zimmermann, editors, The Blackwell Companion to Semantics, Blackwell.

Luca GASPARRI and Diego MARCONI (2016), Word Meaning, in Edward N. ZALTA, editor, The Stanford Encyclopedia of Philosophy, Metaphysics Research Lab, Stanford University, spring 2016 edition, https:

//plato.stanford.edu/archives/spr2016/entries/word-meaning/.

Dirk GEERAERTS (2017), Lexical Semantics, in Oxford Research Encyclopedia of Linguistics, Oxford University Press, doi:10.1093/acrefore/9780199384655.013.29, http://linguistics.oxfordre.com/view/10.1093/acrefore/ 9780199384655.001.0001/acrefore-9780199384655-e- 29.

Eriko KinoshitA, Koji MineshimA, and Daisuke BEKKI (2017), An Analysis of Selectional Restrictions with Dependent Type Semantics, in Setsuya Kurahashi, Yuiko OHTA, Sachiyo Arai, Ken SATOH, and Daisuke BeKKI, editors, New Frontiers in Artificial Intelligence - JSAI-isAI 2016 Workshops, LENLS, HAT-MASH, AI-Biz, JURISIN and SKL, Kanagawa, Japan, November 14-16, 2016, Revised Selected Papers, volume 10247 of Lecture Notes in Computer Science, pp. 19-32, Springer.

Sven LAUER (2004), A Comparative Study Of Current Theories Of Polysemy In Formal Semantics, Master's thesis, Cognitive science Osnabrück - Computational Linguistics, http://cogsci.uni-osnabrueck.de/ CL/.

Bruno MERY and Christian RETORÉ (2017), Classifiers, Sorts, and Base Types in the Montagovian Generative Lexicon and Related Type Theoretical Frameworks for Lexical Compositional Semantics, in Stergios CHATZIKYRIAKIDIS and Zhaohui Luo, editors, Modern Perspectives in Type Theoretical Semantics, pp. 163-188, Springer.

Geoffrey NunBERG (1979), The Non-Uniqueness of Semantic Solutions: Polysemy, Linguistic and Philosophy, 3:143-184.

Geoffrey NUNBERG (1995), Transfers of meaning, Journal of semantics, 12(2):109-132.

James Pustejovsky (1991), The Generative Lexicon, Computational Linguistics, 17(4):409-441.

James Pustejovsky (1995), The generative lexicon, M.I.T. Press.

Aarne RANTA (1994), Type-Theoretical Grammar, Clarendon Press, Oxford.

Livy REAL and Christian RETORÉ (2014), Deverbal semantics and the Montagovian generative lexicon $\Lambda T y_{n}$, Journal of Logic Language and Information, doi:10.1007/s10849-014-9187-y, 10.1007/s10849-014-9187-y. Christian RETORÉ (2014), The Montagovian Generative Lexicon $\Lambda T y_{n}$ : a Type Theoretical Framework for Natural Language Semantics, in Ralph MATTHES 


\section{Robin Cooper, Christian Retoré}

and Aleksy SCHUBERT, editors, 19th International Conference on Types for Proofs and Programs (TYPES 2013), volume 26 of Leibniz International Proceedings in Informatics (LIPICs), pp. 202-229, Schloss Dagstuhl-Leibniz-Zentrum fuer Informatik, Dagstuhl, Germany, ISBN 978-3-939897-72-9, ISSN 1868-8969, doi:10.4230/LIPIcs.TYPES.2013.202.

Göran SundHolm (1986), Proof Theory and Meaning, in Dov GABBAY and Franz GuENTHNER, editors, Handbook of Philosophical Logic, Vol. III, Reidel, Dordrecht.

This work is licensed under the Creative Commons Attribution 3.0 Unported License. http://creativecommons.org/licenses/by/3.๑/ 